\title{
Stress generation and evolution in oxide heteroepitaxy
}

\author{
Aline Fluri ${ }^{*}$ and Daniele Pergolesi ${ }^{\dagger}$ \\ Thin Film and Interfaces Group, Research with Neutrons and Muons Division, Paul Scherrer Institute, 5232 Villigen PSI, Switzerland \\ Alexander Wokaun \\ General Energy Division, Paul Scherrer Institute, 5232 Villigen PSI, Switzerland \\ Thomas Lippert \\ Thin Film and Interfaces Group, Research with Neutrons and Muons Division, Paul Scherrer Institute, 5232 Villigen PSI, Switzerland; \\ Department of Chemistry and Applied Biosciences, Laboratory of Inorganic Chemistry, ETH Zurich, Switzerland; \\ and Molecular Photoconversion Devices Division, International Institute for Carbon-Neutral Energy Research, Kyushu University, Japan
}

(Received 11 December 2017; published 9 March 2018)

\begin{abstract}
Many physical properties of oxides can be changed by inducing lattice distortions in the crystal through heteroepitaxial growth of thin films. The average lattice strain can often be tuned by changing the film thickness or using suitable buffer layers between film and substrate. The exploitation of the full potential of strain engineering for sample or device fabrication rests on the understanding of the fundamental mechanisms of stress generation and evolution. For this study an optical measurement of the substrate curvature is used to monitor in situ how the stress builds up and relaxes during the growth of oxide thin films by pulsed laser deposition. The relaxation behavior is correlated with the growth mode, which is monitored simultaneously with reflection high-energy electron diffraction. The stress relaxation data is fitted and compared with theoretical models for stress evolution which were established for semiconductor epitaxy. The initial stage of the growth appears to be governed by surface stress and surface energy effects, while the subsequent stress relaxation is found to be fundamentally different between films grown on single-crystal substrates and on buffer layers. The first case can be rationalized with established theoretical models, but these models fail in the attempt to describe the growth on buffer layers. This is most probably due to the larger average density of crystalline defects in the buffer layers, which leads to a two-step stress relaxation mechanism, driven first by the nucleation and later by the migration of dislocation lines.
\end{abstract}

DOI: 10.1103/PhysRevB.97.125412

\section{INTRODUCTION}

In epitaxial thin films strain is induced by a lattice mismatch of film and substrate when the thin film adapts to the in-plane lattice parameter of the single-crystalline substrate fully or partially, resulting in coherent or semicoherent interfaces, respectively. Strain is used to induce, inhibit, or modify a range of physical properties in epitaxial metal oxide films. This so-called strain engineering can, for example, be used to tune multiferroic $[1,2]$ properties, the behavior of correlated systems [3], the band gap in metal oxides [4], superconductive properties [5,6], and the thermal [7] and the ionic conductivity [8]. The prerequisite for such studies is a growth platform that provides a suitable lattice mismatch without affecting the characterization of the film of interest. Should no suitable single-crystalline substrate be available, an additional epitaxial layer ("buffer layer") is introduced between film and substrate.

\footnotetext{
*Present address: Molecular Photoconversion Devices Division, International Institute for Carbon-Neutral Energy Research, Kyushu University, Japan.

†daniele.pergolesi@psi.ch
}

Strain-engineering studies typically make use of the thickness dependency of epitaxial strain, which points to the importance of understanding the mechanism of strain generation and relaxation during the growth of epitaxial metal oxide films. In spite of the extensive literature on the effect of strain on material properties, few studies investigate the strain relaxation during the epitaxial growth of oxides [9-13]. In comparison, the strain relaxation in semiconductor epitaxy is very well understood, and many models have been developed and compared to experimental observations [14-21]. The general concept of most models is that with increasing film thickness, the total elastic energy of the film increases. At the "critical thickness" it becomes energetically favorable to introduce crystalline defects, which reduce the strain. From the critical thickness onwards, the average film strain will decrease with increasing thickness through the introduction of more and more crystalline defects [22-25]. The purpose of this work is to discuss for several films, buffer layers, and substrate combinations how theoretical models developed for semiconductor epitaxy can describe the relaxation behavior of epitaxial metal oxide films. The stress evolution, strain $\epsilon$, and stress $\sigma$ being related as $\sigma=E \epsilon$ over the elastic modulus $E$ is monitored in situ during pulsed laser deposition while the acquired data is fit with theoretical models. The models chosen 
to fit or discuss the stress evolution data are the Matthews and Blakeslee equilibrium model ("MB model") [26], the Matthews, Mader, and Light kinetic model ("MML model") [27], a more recently proposed model by Beresford, Lynch, and Chason ("BLC model") [17], and the Cammarata, Sieradzki, and Spaepen model ("CSS model") [28-30]. In the following the models are briefly introduced.

The MB model [26] is based on calculations by Frank and van der Merwe [31]. A dislocation line that continues from the substrate into the film is considered. If the dislocation line in the film migrates parallel to the interface through the film, additional interface dislocations are created that reduce the strain. The calculations evaluate the force balance between forces corresponding to the strain and forces in the dislocation line (i.e., crystallographic constraints keeping the dislocation from moving). As long as the force from strain is smaller, the interface remains defect free. At the critical thickness $h_{c}$, the strain force becomes dominant and it is favorable to introduce interface dislocations by the bowing and elongating of existing threading dislocations. Assuming a perfect epitaxial growth, the strain $\epsilon$ is equal to the lattice misfit $f$ between film and substrate up to the critical thickness ("coherent interface"). Since the stress $\sigma=E \epsilon$, let $F:=E f$. So, according to the MB model the stress remains constant and equal to $F$ up to $h_{C}$. To reduce the number of fitting parameters and because different values for the elastic moduli of film materials can be found in literature, $F$ is assumed to be the largest measured stress value before the film relaxes. Above $h_{c}$, the strain will relax. Assuming that the system is always at equilibrium, i.e., that at each point of the growth, the exact amount of interface dislocations is present to keep the forces equal, the stress as a function of thickness $h$ above $h_{c}$ is described as

$$
\sigma_{\mathrm{MB}}=c_{1} \frac{b}{h}\left(\operatorname{Ln} \frac{h}{b}+1\right) .
$$

For comparing the MB model to the stress data, the elastic constants and the variables describing the dislocation geometry are summarized in the fitting variable $c_{1}$,

$$
c_{1}=E \frac{1-v \cos ^{2} \gamma}{2 \pi(1+v) \cos \eta},
$$

with the Poisson ratio $\nu$, and $\gamma$ and $\eta$ describing the geometry of the dislocation line [26]. The Burgers vector $b$ is the second fitting variable; in oxides, values of $\sim 1-10 \AA$ are reported [32-34].

The MML model [27] is also based on Frank and van der Merwe's work [31]. This model differs from the MB model by not assuming equilibrium, i.e., the system is not necessarily at its energetic minimum during the film growth. Instead, there is an activation energy that has to be overcome for a dislocation line to move. Since the crystal lattice is a periodic system, a dislocation line cannot move by an infinitesimal amount but has to hop from one position to another. This means that the relaxation is not only governed by the lattice mismatch and the type of dislocation, but also, for example, by the temperature $T$. The strain relaxation in $\mathrm{SrTiO}_{3}$ grown on $\mathrm{MgO}$, for example, depends on $T$ [35]. The MML model describes the time $t$ dependent strain, expressing that strain can also relax with time at a constant film thickness. With $t \propto h$ during the film growth, above $h_{c}$ the stress depends on thickness $h$ as

$$
\begin{aligned}
\sigma_{\mathrm{MML}} & =F-\left(F-c_{2} \frac{b}{h} \operatorname{Ln} \frac{h}{b}\right)\left(1-e^{-a h}\right), \\
c_{2} & =E \frac{1-v \cos ^{2} \theta}{8 \pi(1+v) \cos \lambda}, \\
a & =\left(\frac{t}{h}\right) \frac{2 G b^{3} \rho(1+v) \cos \theta \cos ^{2} \lambda D_{0} e^{-U / k_{B} T}}{(1-v) k_{B} T},
\end{aligned}
$$

where $\theta$ and $\lambda$ describe the dislocation geometry, $\rho$ is the number of dislocation lines per area, $G$ the shear modulus, and $k_{B}$ the Boltzmann constant. $D_{0} e^{-U / k_{B} T}$ is the diffusion coefficient of the dislocation core with an activation energy $U$. For $a h \gg 1$ the formula expresses equilibrium, and if additionally $h \gg b, \sigma_{\mathrm{MML}}$ approximates $\sigma_{\mathrm{MB}}$. The parameter $a$ depends on the deposition rate $h / t$, because the model originally describes a time-dependent relaxation but has been modified here to reflect the thickness dependence during the growth.

In the two models above, very specific dislocation geometries are assumed, even though more than one type of dislocation may exist and the possibility of dislocation interaction is not taken into account [22-25]. They further assume a coherent layer-by-layer growth. The growth mode influences the effective strain in a thin film, since the formation of islands partially relieves the average strain as islands are more free to expand or contract $[13,36,37]$. Additionally, the nucleation of dislocations and thereby the strain relaxation is facilitated where adjacent islands merge and for large lattice misfit values $[22,25]$.

Beresford, Lynch, and Chason introduced a kinetic model (BLC model) where the rate-limiting step for the relaxation is not the migration of dislocation lines but the nucleation of dislocations, e.g., for an island growth mode. The reduction in strain is set to $f-\epsilon=b /(2 \times$ average dislocation spacing $)$, and the factor 2 arises from the assumed dislocation geometry. Based on the nucleation rate and the number of potential nucleation sites per surface area, a differential equation and an approximate solution for the strain relaxation above $h_{c}$ is deduced (details are shown in Appendix A).

Other factors that can influence the stress evolution are the surface stress and the surface energy, which are taken into account in a modified MB model proposed by Cammarata, Sieradzki, and Spaepen for a semicoherent interface (CSS model) [28-30]. Based on the Gibbs formalism [38], the surface energy is defined as the reversible work per unit area to create a new surface in a solid, and the surface stress is defined as the reversible work per unit area needed to elastically stretch an existing surface. In the CSS model, the inclusion of surface effects leads to the term (surface energy-surface stress) $/ h:=$ $d / h$ being added to the MB expression of stress [Eq. (1)]:

$$
\sigma_{\mathrm{CSS}}=\sigma_{\mathrm{MB}}+\frac{d}{h} .
$$

In Fig. 1, the strain relaxation according to the $\mathrm{MB}$, the MML, and the CSS model is shown. For very large $a$, MML approaches roughly the shape of the MB model. For smaller $a$, the relaxation becomes less pronounced. 


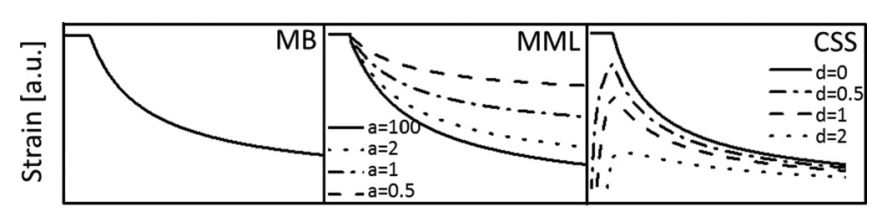

Thickness [a.u.]

FIG. 1. Models for strain relaxation. The strain relaxation according to the MB, the MML, and the CSS model is shown at arbitrary scales. The MML and the CSS model are shown for different values of the parameters $a$ and $d$, respectively. $a$ summarizes various parameters that influence the stress relaxation, such as the temperature or the number of dislocation lines [see Eq. (2)]. $d$ is the difference between the surface energy and the surface stress [see Eq. (3)].

Here we report the experimental study of the stress relaxation monitored in situ for six different cases of epitaxial oxide thin-film growth on single-crystal substrates and on buffer layers. The comparative analysis of the experimental data using the above-described theoretical models is presented, and the fundamentally different relaxation behavior for films grown with or without a buffer layer is discussed.

\section{EXPERIMENTAL}

Thin films of $\mathrm{SrTiO}_{3}$ (STO), Sm-doped $\mathrm{CeO}_{2}$ (SDC), $\mathrm{BaZrO}_{3}$ (BZO), Ce-doped BZO (BZC), Y-doped BZO (BZY) are fabricated by pulsed laser deposition (PLD) in a UHV chamber by Twente Solid State Technology [39]. The chamber is equipped with reflection high-energy electron diffraction (RHEED) system and a multibeam optical stress sensor (MOSS), both from k-space Associates, Inc. [40]. A thin film growing under tensile or compressive stress exerts a force parallel to the substrate surface that bends the substrate. The working principle of the MOSS is based on Stoney's equation, which expresses the relation between film stress $\sigma$ and substrate curvature $1 / \rho$,

$$
\sigma h=\frac{h_{s}^{2} Y}{6(1-v)} \frac{1}{\rho},
$$

with $h$ being the film, $h_{s}$ the substrate thickness, and $Y$ and $v$ the Young's modulus and Poisson ratio of the substrate. The MOSS measures the curvature using the deflection of parallel laser beams [41]. To monitor the stress, a $3 \times 3$ array of laser beams is used. $10 \mathrm{~mm} \times 10 \mathrm{~mm} \times 0.5 \mathrm{~mm}$ (110)-oriented $\mathrm{NdGaO}_{3}$ (NGO) and (100)-oriented $\mathrm{MgO}$ single-crystal substrates provided by Crystec $\mathrm{GmbH}$ are used. Young's modulus and the Poisson ratio of the substrates used in this work are for NGO $Y=239 \mathrm{GPa}$ and $v=0.29$ [42] and for $\mathrm{MgO}, Y=215 \mathrm{GPa}$ and $v=0.26$ [43]. Sintered pellets are ablated by a $248-\mathrm{nm}$ $\mathrm{KrF}$ excimer laser (Lambda Physics $\mathrm{GmbH}$ ) with a pulse length of $25 \mathrm{~ns}$ and a repetition rate of $4 \mathrm{~Hz}$. The sample holder is designed for the substrates to bend freely and the samples are heated from the back. A more detailed description of this setup can be found in a previous publication [44]. Since the substrates are transparent to infrared radiation, the back side is sputter coated with $\sim 200 \mathrm{~nm} \mathrm{Pt}$ ( $40 \mathrm{~W}, 4 \mathrm{~min}, 2 \times 10^{-2} \mathrm{mbar}$ Ar, target-substrate distance $4 \mathrm{~cm}$ ) to ensure thermalization of the infrared radiation. The sample temperature is read out with a pyrometer, assuming an emissivity of 1 as the back side of the substrate coated with $\mathrm{Pt}$ is rough and the substrate and film are transparent. STO and SDC films are grown at a temperature of $650^{\circ} \mathrm{C}$ with a target to substrate distance of $5 \mathrm{~cm}$ and a laser fluence of $1.2 \mathrm{~J} / \mathrm{cm}^{2}$ in a oxygen partial pressure of $5 \times 10^{-2}$ mbar. For the STO and BZO buffer layers the same conditions, but a temperature of $750{ }^{\circ} \mathrm{C}$ were used as in [44]. BZY and BZC are grown at a temperature of $750{ }^{\circ} \mathrm{C}$ with a target-to-substrate distance of $6 \mathrm{~cm}$, a laser fluence of $2.8 \mathrm{~J} / \mathrm{cm}^{2}$, and an oxygen partial pressure of $1 \times 10^{-1} \mathrm{mbar}$ as described in [45]. All samples are cooled under growth pressure with $20^{\circ} \mathrm{C} / \mathrm{min}$. For each material, the growth rate was calibrated with $\mathrm{x}$-ray reflectometry.

The theoretical models were fitted to the experimental data with WOLFRAM MATHEMATICA, using the built-in "NonlinearModelFit" function.

\section{RESULTS AND DISCUSSION}

For this study the stress evolution is monitored in situ for the following couplings of thin-film materials and substrates: $\mathrm{SrTiO}_{3}$ (STO) films on $\mathrm{NdGaO}_{3}$ (NGO) substrates, $15 \%$ Smdoped $\mathrm{CeO}_{2}$ (SDC) films on NGO substrates and on $\mathrm{MgO}$ substrates with double buffer layers of $\mathrm{BaZrO}_{3}$ (BZO) and STO, $20 \%$ Y-doped $\mathrm{BaZrO}_{3}$ (BZY) films on $\mathrm{MgO}$ substrates with buffer layers of $40 \%$ Ce-doped $\mathrm{BaZrO}_{3}$ (BZC), and finally, BZC films on $\mathrm{MgO}$ and BZY-buffered $\mathrm{MgO}$ substrates. SDC has the cubic fluorite crystal structure, while BZY is a cubic perovskite. Also STO, BZO, and BZC are cubic perovskites. Concerning the two substrates, $\mathrm{MgO}$ has the rocksalt structure and NGO the pseudocubic perovskite structure. The sample design is summarized in Table I. Table I includes the expected epitaxial relations and the lattice mismatch between the films and the substrates or the films and the upper buffer layers.

SDC and BZY are an oxygen-ion and a proton-conducting oxide, respectively. The most important technological application of these two materials is as electrolyte in solid oxide fuel cells and electrolyzers [46]. To study the influence of tensile strain on the ion conductivity $[8,47]$, a double buffer layer of $5 \mathrm{~nm} \mathrm{BaZrO} 3(\mathrm{BZO})$ and $5 \mathrm{~nm} \mathrm{STO}$ on (001)MgO substrates (SB-MgO) was used for SDC [44,48] and a 30-nm buffer layer of $40 \% \mathrm{Ce}$-doped $\mathrm{BaZrO}_{3}$ (BZC) on (001)MgO substrates was used for BZY [45]. The growth of the BZC layer itself and the inversion of film and buffer layer [BZC on a 10-nm BZY buffer layer on (001) $\mathrm{MgO}$ ] are discussed, too (cases 5 and 6). Cases 3, 4 , and 6 are reproduced data sets from previous studies [44,45].

\section{A. Structural characterization and stress evolution of different epitaxial metal oxide thin films}

The films are in and out of plane epitaxially oriented. $\omega / 2 \theta$ scans shown in Fig. 2(a) demonstrate that all films are out-of-plane (100) epitaxially oriented. For the BZC films on $\mathrm{BZY}-\mathrm{MgO}$, the (011) reflection is also visible with roughly the same intensity as the (001) reflection. Powder diffraction studies show that the (011) reflection in BZC has a 2000 times higher line strength than the (001) reflection [49]. This means that the (011) reflection is truly minor. It likely results from the sample edge and will be ignored for the discussion here. Reciprocal space maps (RSMs) were acquired to demonstrate the in-plane orientation of the films. Figures 2(b) and 2(c) show 
TABLE I. Sample design. The expected epitaxial relation, the lattice mismatch, and the total film thickness are indicated. STO-BZO will be abbreviated as SB. The lattice mismatch is calculated considering the lattice parameter of the unstrained film material and that of either the substrate or the upper buffer layer. Depending on the degree of relaxation of the buffer layer, the effective lattice mismatch may differ from the theoretical value. In such a case the effective lattice mismatch, as determined by XRD, is indicated in brackets.

\begin{tabular}{|c|c|c|c|c|c|c|}
\hline & Substrate & Buffer layer & Film & Epitaxial relations & $f[\%]$ & $h[\mathrm{~nm}]$ \\
\hline 1 & NGO & & STO & $\begin{array}{l}\operatorname{STO}(100) \| \operatorname{NGO}(1-10) \\
\text { STO }(010) \| \operatorname{NGO}(001) \\
\text { STO }(001) \| \operatorname{NGO}(110)\end{array}$ & $\begin{array}{l}-1.28 \\
-1.04\end{array}$ & 102 \\
\hline 2 & NGO & & SDC & $\begin{array}{l}\operatorname{SDC}(1-10) \| \text { NGO }(1-10) \\
\operatorname{SDC}(110) \| \text { NGO }(001) \\
\text { SDC(001)\|NGO(110) }\end{array}$ & $\begin{array}{l}0.64 \\
0.39\end{array}$ & 178 \\
\hline 3 & $\mathrm{MgO}$ & STO-BZO (SB) & SDC & $\begin{array}{l}\operatorname{SDC}(110) \| \operatorname{STO}(100) \\
\operatorname{SDC}(001) \| \operatorname{STO}(001)\end{array}$ & $1.52(1.5-2)$ & 81 \\
\hline 4 & $\mathrm{MgO}$ & $\mathrm{BZC}$ & $\mathrm{BZY}$ & $\begin{array}{l}\mathrm{BZY}(100) \| \mathrm{BZC}(100) \\
\mathrm{BZY}(001) \| \mathrm{BZC}(001)\end{array}$ & $1.47(0.9)$ & 110 \\
\hline 5 & $\mathrm{MgO}$ & $\mathrm{BZY}$ & $\mathrm{BZC}$ & $\begin{array}{l}\mathrm{BZC}(100) \| \mathrm{BZY}(100) \\
\mathrm{BZC}(001) \| \mathrm{BZY}(001)\end{array}$ & $-1.45(-1.5)$ & 117 \\
\hline 6 & $\mathrm{MgO}$ & & $\mathrm{BZC}$ & $\begin{array}{l}\mathrm{BZC}(100)|| \mathrm{MgO}(100) \\
\mathrm{BZC}(001)|| \mathrm{MgO}(001)\end{array}$ & -1.70 & 72 \\
\hline
\end{tabular}

the RSMs of STO and SDC films on NGO (cases 1 and 2 in Table I). The symmetric and asymmetric reflections of the STO films on NGO show a double peak [Figs. 2(a) and 2(b)], indicating nonuniformly strained films. The RSMs of the SDC film on SB-MgO (case 3 in Table I), as well as of the doped-BZO films on $\mathrm{MgO}$ (case 4 to 6), show uniform strain and are reported in our previous studies [44,45].

The in situ MOSS measurements of the stress evolution in the films are shown in Fig. 3. For each material, the curvature [Fig. 3(a)] was monitored in situ until it became constant. After the growth was stopped, the curvature remained constant, which means that there is no indication of further relaxation. The stress [Fig. 3(b)] is calculated using Stoney's equation

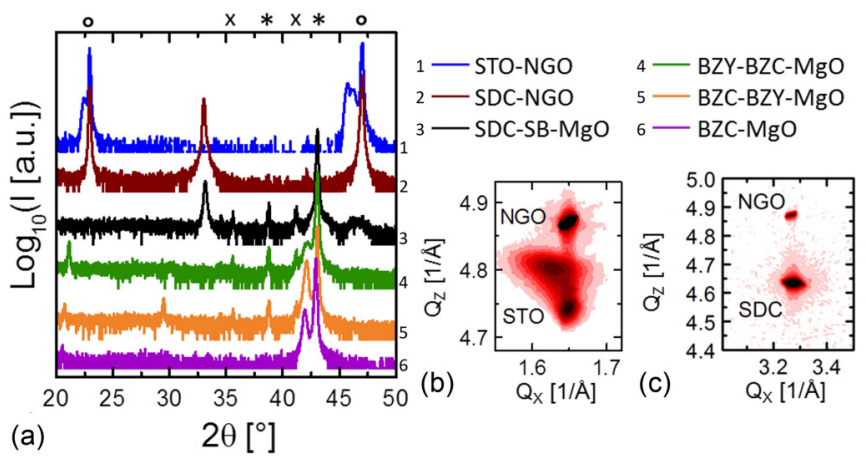

FIG. 2. XRD analysis of crystallographic orientations. The $\omega / 2 \theta$ scans (a) show that the films are out-of-plane epitaxial oriented [with a very minor $(110)$ phase for $\mathrm{BZC}$ on $\mathrm{BZY}-\mathrm{MgO}] . \times$ indicates a background peak. $\circ$ and $*$ indicate the substrate reflections of NGO and $\mathrm{MgO}$, respectively. At around $38^{\circ}$ the $\mathrm{Cu} \mathrm{K} \beta$ peak of $\mathrm{MgO}$ is sometime visible and marked also with $*$. The RSM in (b) shows the STO (103) reflection and the NGO (332) reflection the STO film on NGO $\left[Q_{X}\|\mathrm{NGO}(001)\| \mathrm{STO}(100)\right.$ and $\left.Q_{Z}\|\mathrm{NGO}(110)\| \mathrm{STO}(001)\right]$. The RSM in (c) shows the (224) reflection of SDC and the (334) reflection of $\mathrm{NGO}\left[Q_{X}\|\mathrm{NGO}(001)\| \mathrm{SDC}(110)\right.$ and $\left.Q_{Z}\|\mathrm{NGO}(110)\| \operatorname{SDC}(001)\right]$.
Eq. (4). In all cases the sign of the stress is consistent with the strain measured by x-ray diffraction (XRD) and with the lattice mismatch between film and growth platform: tensile lattice distortions imply a positive curvature and a positive stress value and vice versa for compressive lattice distortions.

The MOSS is used here rather as a qualitative than a quantitative tool. Directly comparing the in situ stress measurements by MOSS and the ex situ strain measurements by XRD is challenging since various values for the elastic constants are reported in literature and the two measurements are performed at very different temperatures. The materials used here have very similar thermal expansion coefficients so that this point can be neglected. The thermal strain would be below $0.1 \%$, which is within the error of our strain measurements. Challenges when directly comparing stress values from MOSS and strain values from RSM were discussed earlier [44]. Furthermore, it is not possible to follow the film stress when changing
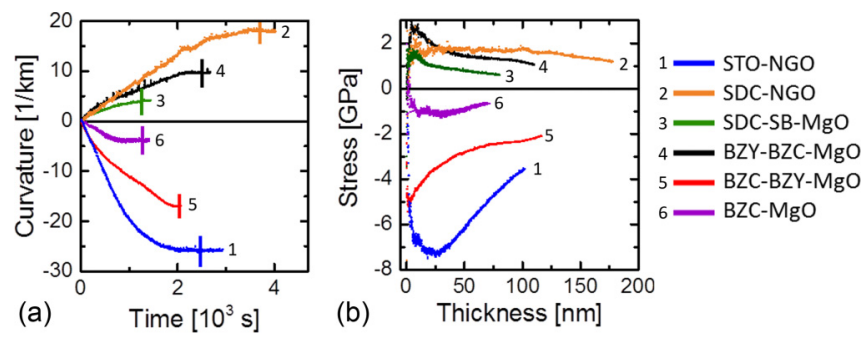

FIG. 3. Substrate curvature and stress measurements. The curvature evolution with time (a) and the stress evolution with thickness (b) is shown for growth of different oxide materials. The data in (b) is calculated from the Stoney equation [Eq. (4)], i.e., the average stress of the film during the growth process. The samples are labeled in the legend as film-(buffer layer-)substrate and are listed in the order cases 1-6 (Table I). A vertical line indicates in (a) where the growth was stopped. Films of BZC, STO, and SDC were grown on single-crystalline substrates. Films of BZC, BZY, and SDC were grown on epitaxial buffer layers. 


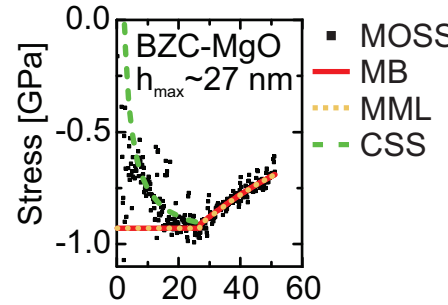

(a) Thickness [nm]

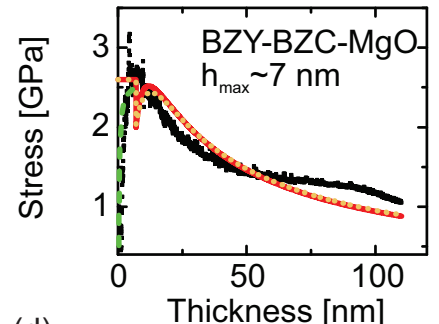

(d)

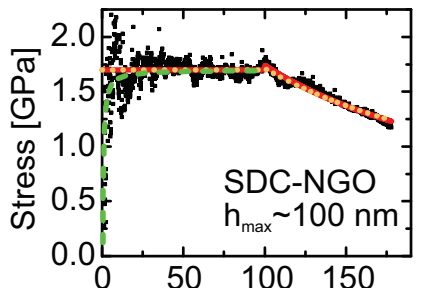

(b)

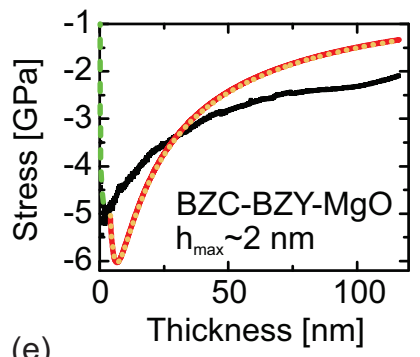

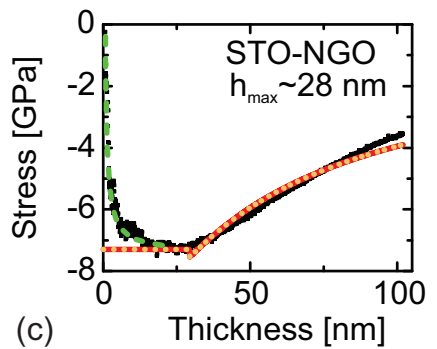

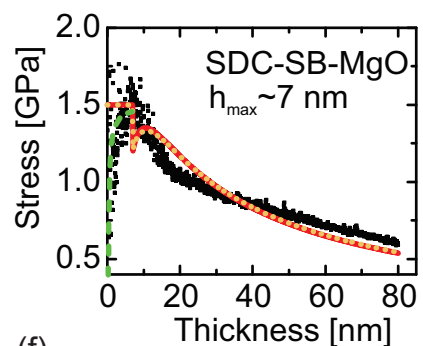

(f)

FIG. 4. Comparison of stress measurement and models. The models [Eqs. (1), (2), and (3)] are fit to the stress data shown in Fig. 3. The MB and MML models are fit for $h>h_{\max }$. Below $h_{\max }$, the stress would be constant according to theses models. The CSS model is fit for $h<h_{\max }$. The values used as $h_{\max }$ are indicated for each case. Fitting parameters are listed in Appendix C.

the temperature, because large fluctuations result, e.g., from the thermal expansion of the heater stage, which changes geometrical parameters in the curvature measurement.

The stress measured by the MOSS is the average value over the entire film thickness, and therefore there are limitations in analyzing inhomogeneous lattice distortions or separating surface effects. Unless otherwise stated, we consider in this work the average stress and also strain as we can identify it by the in situ measurements.

\section{B. Discussion of MOSS measurements using theoretical models}

All the stress evolution curves show that $|\sigma|$ reaches a maximum and decreases above a certain thickness $h_{\max }$ with increasing thickness, as shown in Fig. 4. For $h<h_{\max }$ the stress approaches zero when the thickness approaches zero. This disagrees with the MB and MML models where the stress would be constant up to the critical thickness. Figures 4(a), 4(c), 4(d), and 4(f) clearly show the large deviation of the experimental data from these two models. This feature is significant, since the baselines recorded for all cases before the start of the growth are in the MOSS measurement intrinsically fluctuating around $1 / \rho=0 \mathrm{~km}^{-1}$ (more details in Appendix B). The growth of SDC on NGO agrees best with the MB model [Fig. 4(b)], while for BZC on BZY-MgO [Fig. 4(e)] the value of $h_{\max }$ is very small (a few nanometers). Figure 4 clearly shows that only the CSS model can describe the stress evolution at the initial stage of the growth well, i.e., below $h_{\max }$. This observation strongly suggests that surface effects dominate the initial stage of the growth. The values obtained for $d$ [the parameter in Eq. (3) accounting for the difference between surface energy and surface stress] are in the range of a few GPa nm, which is on the same scale as was predicted for metals [28].

We consider now the stress evolution for $h>h_{\max }$. For the growth of BZC on MgO and SDC on NGO [Figs. 4(a) and 4(b)], the MB and MML models can be used to fit the experimental data, while a significant deviation appears for the growth of STO on NGO shown in Fig. 4(c). Finally, for all films grown on buffer layers, the results of the MB and MML models are far off. A much better fitting can be obtained by applying the BLC model [17], which consists of a differential equation for the strain as a function of thickness. An approximate solution was presented [17] which can model all six stress evolution curves above $h_{\max }$ within the accuracy of the measurement noise. However, this may simply be due to the fact that four fitting parameters are used. A polynomial equation with the same number of fitting parameters (i.e., third order) can actually fit the data as well as the BLC model. In Appendix C, all fitting results are listed in Tables II-V and the comparison of the BLC model and the polynomial fit is shown in Fig. 7.

We also note that the MB and MML models produce a basically equivalent fit. By comparing the fundamental assumptions of the two models, this finding can be taken as an indication that the elastic energy and the formation energy for dislocations are always at equilibrium. This hypothesis is supported by the constant curvature after the end of the growth (Fig. 3). Such a behavior is also known for semiconductors and is in disagreement with kinetic models where the strain is time-dependent, like the MML model or also the model proposed by Dodson and Tsao [17,21,50]. Therefore it seems that the experimental data here cannot be described with the MML model. It is possible, though, that on longer time scales such as during cooling, the strain relaxes further to some extent. The general correlation of the in situ, real-time observation of the stress during the growth and the ex situ strain measurements after cooling certainly deserves a dedicated future investigation.

\section{Stress relaxation and growth mechanism}

Reflection high-energy electron diffraction was used to investigate the growth mode of the films in combination with the 
MOSS measurements of the stress evolution. We discuss next the different stress relaxation scenarios, starting with the case of thin films deposited directly on single-crystal substrates. The RHEED patterns acquired during the growth of the SDC films on $\mathrm{NGO}$, as well as those of the BZC films on $\mathrm{MgO}$, showed the typical features of the islandlike growth (Volmer-Weber and Stranski-Krastanov growth mode). Even though the MB model was set up under the assumption of a layer-by-layer (Frank-van der Merwe) growth mode, it still appears to describe the growth of SDC on NGO and BZC on MgO [Figs. 4(a) and 4(b)] well. The substrate curvature [Fig. 3(a)] increases with a roughly steady slope [i.e., constant stress, see Eq. (4)]. Continuing the growth, the slopes of the curvature vs time curves decrease and become gradually constant while the stress decreases [Fig. 3(b)]. A constant curvature with increasing thickness means that the total elastic energy of the film remains constant, which corresponds to the onset of relaxation in the MB model. Every increase in elastic energy, which the increasing thickness would cause, is immediately compensated by the introduction of more dislocations. However, the fitted values of the Burgers vectors $b$ are about a factor of 10-100 larger than the few $\AA$ typically reported for oxides [32-34]. Assuming $b=5 \AA$, $\sigma\left(h_{c}\right)=F$, and $h_{c}=h_{\max }$ in the MB model, the stress would relax much faster and, a few nanometers above $h_{\max }$ it would be much smaller than the measured values. Looking at Eq. (1), $b$ is basically scaling the thickness (or time) axis, meaning that an overly large value of $b$ can imply that the relaxation process is occurring slower than the MB model would predict. A reason for such a delay is that the movement of threading dislocations can be blocked or hindered by interactions among them or by other crystalline defects [22,25]. For example, Freund's blocking criterion [16,51] was used to explain the strain relaxation of $\mathrm{MgO}$ grown on $\mathrm{Fe}$ [32].

The SDC films on NGO have by far the largest $h_{\max }$ while the lattice mismatch is the smallest, which is perfectly in line with the MB model. Also, in thin films (30 nm $\left.\ll h_{\max }\right)$, the strain value was only slightly below the lattice mismatch [44]. Likely, a few interface dislocations nucleated at the interface due to the island growth mode, allowing the average strain to be slightly reduced as compared to the ideal case of a coherent interface. Within the framework of the BLC model, the island growth mode observed for SDC grown on NGO could produce a steady dislocation nucleation rate so that the film gradually relaxes. It is generally thought that islands expand or contract to accommodate strain, resulting in larger local distortions in the area where two islands merge, which facilitates the nucleation of dislocations [17,22,25]. Nevertheless, the stress in SDC remains constant up to a large thickness, which means that the island growth mode did not cause a steady relaxation. In this film, the likelihood of dislocation nucleation is reduced due to the small lattice mismatch, which leads to small local distortions between islands. The island growth mode in SDC is also likely not driven by strain but results because the (111) facets of SDC have a lower surface energy than the (001) facets [52].

Let us consider now the film of BZC grown on $\mathrm{MgO}$ substrate. As compared to the previous case, SDC on NGO, the stress has the opposite sign, i.e., the in-plain strain state of the film is compressive instead of tensile. It is worth noticing that the overall relaxation behavior is very similar in the two cases.
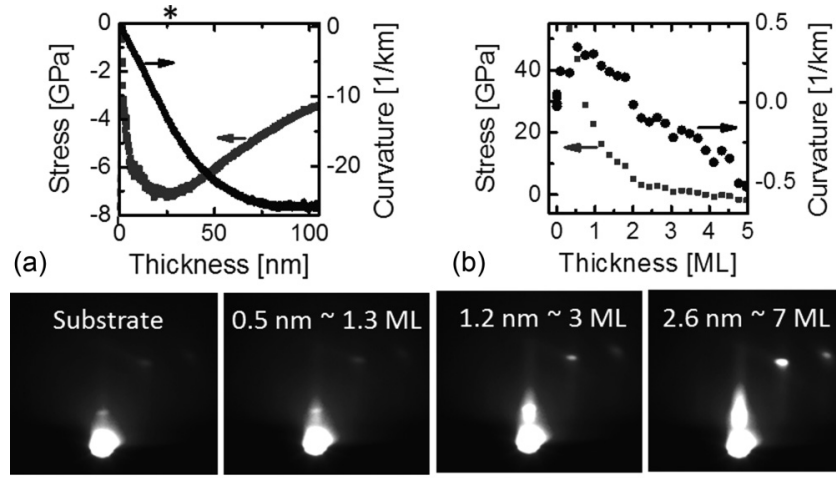

(b)
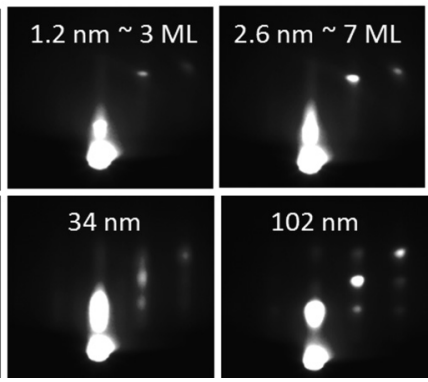

FIG. 5. Correlation of MOSS and RHEED results for the STO growth on NGO. The stress and curvature evolution measured by MOSS (a) [the initial stage of the growth magnified in (b)] and the RHEED pattern (c) are shown. The indicated thickness in nanometers is calculated from the growth rate determined from a $\sim 40 \mathrm{~nm} \mathrm{STO}$ film, and the indicated number of monolayers (ML) is the thickness divided by the lattice constant of STO $(\sim 3.9 \AA)$. The reciprocal space map shows the $\mathrm{NGO}(332)$ and the $\mathrm{STO}(103)$ reflections. $Q_{X}\|N G O(001)\| S T O(100)$ and $Q_{Z}\|N G O(110)\| S T O(001)$. The * in (a) indicates the thickness at which RHEED showed the transition between the layer-by-layer and island growth modes.

However, for $\mathrm{BZC}$ on $\mathrm{MgO}$ the relaxation starts earlier and, despite the larger lattice mismatch between film and substrate, smaller absolute stress [Fig. 3(b)] and strain (Table I) values are reached below $h_{\max }$. As suggested previously [45], the larger lattice misfit may favor the nucleation of dislocations between islands, thus facilitating the stress relaxation.

The third example of film growth directly on a single-crystal substrate is given by the STO films grown on NGO [Fig. 4(c)]. With the selected deposition parameters, the STO films initially grow in a layer-by-layer mode with a transition to an island growth mode after $\sim 26 \mathrm{~nm}$ [Fig. 5(a)], which becomes more pronounced as the growth continues. The thickness at which the RHEED showed the change of the growth mode coincides with the onset of stress relaxation as observed by MOSS, i.e., with the reduction of the compressive stress after $h_{\max } \approx$ $28 \mathrm{~nm}$, as shown in Figs. 4(c) and 5(b). It is interesting to see how the change of the morphology of the surface at a nanometric level correlates directly to the macroscopic change of the wafer curvature detectable by optical measurements. A similar behavior was reported, for example, also for the growth of $\mathrm{CeO}_{2}$ on $12 \%$ Y-stabilized zirconia substrates $(f \approx 5 \%)$ [53] and of $\mathrm{LaAlO}_{3}$ grown on $\mathrm{STO}(f \approx 3 \%)$ substrates [54].

The BLC model describes exactly this kind of coupling between the relaxation and the growth mode. Further, this change in growth mode can now explain the nonhomogeneous strain distribution found by XRD [Fig. 2(b)]. When islands start to form, the nucleation rate for dislocations increases and as a result, the part of the film below $h_{\max }$ remains strained while the rest relaxes. 
It is interesting to note that during the growth of the first few nanometers the substrate curvature (and as a consequence, the stress) does not change monotonically with the expected sign, but the substrate bends first in the opposite direction during a number of laser pulses that correspond on average to $\sim 1$ monolayer [Fig. 5(b)]. The significance of this feature is discussed in Appendix B. Calculating the stress from the curvature data [Eq. (4)] yields a very large tensile strain during the first 1-2 monolayers, which can be described well by the CSS model. The same feature was observed for the growth of STO on (001)Pd single crystals [55], and it was suggested to result from the bonds in an incomplete unit cell being different from those in the bulk. Consequently, a different lattice mismatch (and therefore strain state) arises.

\section{Different relaxation behavior with and without buffer layer}

Comparing the films grown directly on the single-crystal substrates with those grown on buffer layers [Figs. 3(d)-3(f)], it can be seen that the stress evolution is fundamentally different. The curvature data of films directly grown on substrates has an approximately constant slope up to $h_{\max }$, whereupon the curvature becomes constant with time in accordance with the MB model. During the growth on buffer layers, the slope of the curvature also changes at $h_{\max }$, which is reflected by a steep drop in the stress right above $h_{\max }$, but the curvature keeps changing with time up to a significantly larger thickness. This can be seen well in Fig. 3(a) when comparing BZC-BZY$\mathrm{MgO}$ (red) with STO-NGO (blue) or when comparing SDC$\mathrm{SB}-\mathrm{MgO}$ (green) with SDC-NGO (orange).

The buffer layers likely contain more crystalline defects than a single-crystalline substrate, and defects are sites for dislocation nucleation. Within the framework of the BLC model, we hypothesize that it is the larger average density of dislocations that leads to the faster relaxation at the beginning of the growth (the steep drop). The larger density of defects as compared to the films grown directly on substrates is likely outgrown gradually, and the slope of the curvature becomes approximately constant [Fig. 3(a)]. With the substrate curvature, also the total elastic energy of the film remains constant. The stress relaxation through the migration of dislocation lines described in the MB model now likely becomes the dominant relaxation process.

Finally, it is interesting to compare the cases 4-6 in Table I that involve different combinations of BZY and BZC films on $\mathrm{MgO}$ substrates. Let us consider first the two materials grown directly on $\mathrm{MgO}$. It has been shown that $\mathrm{BZY}$ can form an almost coherent interface with $\mathrm{MgO}$, implying that below a certain thickness the in-plain compressed lattice parameter of BZY equals that of MgO $[45,48]$. Instead, a thin film of BZC with the same thickness (about $30 \mathrm{~nm}$ ) shows an in-plane lattice parameter very similar to fully relaxed BZY [45]. As a consequence, even using the same substrate and the same thicknesses for the film and buffer layer, when BZY grows on BZC (case 4), the developed stress is significantly smaller than when BZC grows on BZY (case 5). This is shown in Fig. 3, which highlights once more the potential of MOSS as a powerful in situ diagnostics of the evolution of stress in thin films.

Second, we consider now the BZC films grown on a BZY buffer layer (case 5) and directly on $\mathrm{MgO}$ (case 6). As mentioned above, the BZY buffer layer used in case 5 has about the same in-plane lattice parameter as the $\mathrm{MgO}$ substrate. Thus the lattice mismatch between the BZC films and the two growth platforms of cases 5 (BZY-MgO) and $6(\mathrm{MgO})$ are nearly the same. Nevertheless, Fig. 3 evidently shows that the stress in $\mathrm{BZC}$ grown on $\mathrm{MgO}$ is much smaller than that of BZC grown on BZY-MgO, even though in the latter case the film grew on a buffer layer that typically contains more crystalline defects which facilitate the stress relaxation. Since the interfacial lattice misfit is the same in the two cases, we speculate that it is the difference of the crystal structure, perovskite for BZC and $\mathrm{BZY}$ and rocksalt for $\mathrm{MgO}$, which makes the stress relaxation easier in case 6 as compared to case 5 .

\section{CONCLUSION}

The stress relaxation behavior was monitored in situ by MOSS and RHEED for several cases of metal oxide epitaxy. The results were rationalized by fitting the experimental data with established theoretical models. The initial stage of the growth appears to be governed by surface effects such as surface energy and stress. The subsequent stress relaxation was found to be different for films grown on single-crystal substrates and films grown on buffer layers. In the first case, the stress evolution is explained with theoretical models established for semiconductor epitaxy. For example, the stress is preserved up to a larger thickness if the lattice mismatch is smaller. Moreover, the onset of relaxation correlates with the transition from a layer-by-layer to an island growth mode. In the second case, i.e., for films grown on buffer layers, the stress likely relaxes through two different mechanisms. We suggest that the initially steep decrease of the stress is dominated by the nucleation of dislocations, while with increasing the thickness the migration of dislocation lines begins, causing a further reduction of the stress. The different relaxation behavior of films grown on buffer layers and on single-crystalline substrates is most probably due to the different crystalline quality and it is important to consider in multilayer heteroepitaxy, which is often employed to investigate strain effects. The combination of MOSS and RHEED represents an invaluable diagnostic tool to investigate the stress relaxation in thinfilm epitaxy. Indeed, the complementary information gained from combining in situ substrate curvature measurements and surface electron diffraction has significantly contributed to understanding the stress relaxation mechanisms.

\section{ACKNOWLEDGMENT}

The research leading to these results has received funding from the Swiss National Foundation for Science (SNFS) under Grant Agreement No. 200021-126783.

\section{APPENDIX A: STRESS RELAXATION ACCORDING TO THE BLC MODEL}

In the Beresford, Lynch, and Chason model [17], the reduction in strain is set to $f-\epsilon=b /(2 \times$ average dislocation spacing), and the factor 2 arises from the dislocation geometry. Let $\epsilon_{\text {norm }}:=\epsilon / f$. The model now yields for 
thickness $h \gg h_{c}$,

$$
\begin{aligned}
\epsilon_{\text {norm }}= & \left\{\epsilon_{0, \text { norm }}^{-\alpha}+2 f \alpha\left[\frac{\kappa}{b}\left(h-h_{0}\right)-\eta b\left(\frac{n_{s 0}}{b}-\frac{\kappa}{b}\right)\right.\right. \\
& \left.\left.\times\left(1-e^{-\left(h-h_{0}\right) /(\eta b)}\right)\right]\right\}^{-1 / \alpha},
\end{aligned}
$$

where $h_{0}$ is the thickness where the relaxation starts, and $\epsilon_{0, \text { norm }}=\epsilon_{\text {norm }}\left(h_{0}\right)$. The meaning of parameters $\alpha, \kappa, \eta$, and $n_{s 0}$ can be found in Ref. [17]; the purpose here is to rewrite the expression above to obtain $\sigma(h)$.

With $\sigma=E \epsilon=E f \epsilon_{\text {norm }}$, setting $F=E f \epsilon_{0, \text { norm }}$ and $h_{0}=$ $h_{\max }$ in relation to the stress measurements, as well as redefining unknown variables as $\eta^{\prime}=\eta b, \kappa^{\prime}=2 E^{-\alpha} f^{1-\alpha} \alpha \kappa / b$, and $n=2 E^{-\alpha} f^{1-\alpha} \alpha \eta b\left(n_{s 0} / b-\kappa / b\right)$, it follows that

$\sigma(h)=F\left\{1+F^{\alpha}\left[\kappa^{\prime}\left(h-h_{\max }\right)-n\left(1-e^{-\left(h-h_{\max }\right) /\left(\eta^{\prime}\right)}\right)\right]\right\}^{-1 / \alpha}$.

\section{APPENDIX B: SIGNIFICANCE DURING INITIAL STAGE OF GROWTH}

The baseline recorded before the growth start is intrinsically at zero (Fig. 6), since the MOSS measurement is very sensitive to changes in the curvature but cannot measure the absolute value of curvature. This is explained through the working principle of the MOSS: parallel laser beams are reflected by the substrate and recorded with a CCD camera. When a strained film grows, the substrate bends and the distance $D$ between the reflected beams changes. The relative change in distance as compared to the initial value $D_{0}$ is proportional to the curvature over geometric parameters of the measurement setup: $\left(D-D_{0}\right) / D_{0} \propto 1 / \rho$ [41]. Consequently, any feature at the beginning of the growth which is distinguishable from the noise of the baseline is attributed to the stress evolution of the film.

If the feature that is assigned to the CSS model $[\sigma \rightarrow 0$ for $h(>0) \rightarrow 0$ ] would only be present in one case, it might just be a random divergence through the noise in the initial stage of the growth. However, it occurs in none of the cases that $\sigma \rightarrow \infty$ for $h(>0) \rightarrow 0$, hence we do not consider this feature random.

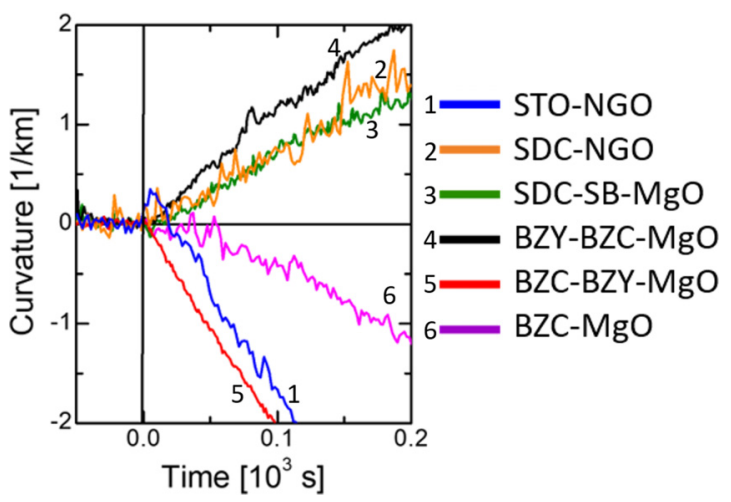

FIG. 6. Initial stage of the growth. Zoom in to time around the growth start in Fig. 3.
The special case in the STO film on NGO of the "inverse" stress state in the first few monolayers is also clearly a significant feature, as can be seen in Fig. 6 .

\section{APPENDIX C: FITTING PARAMETERS}

\section{Matthews and Blakeslee Model}

TABLE II. Fit Parameters for Matthews and Blakeslee model [26]. Additionally, the maximal stress value $F$ and the thickness $h_{\max }$ after which the stress decreases are listed.

\begin{tabular}{lcccc}
\hline \hline Sample & $c_{1}[\mathrm{GPa}]$ & $b[\mathrm{~nm}]$ & $F[\mathrm{GPa}]$ & $h_{\max }[\mathrm{nm}]$ \\
\hline SDC on NGO & 2.70 & 28.80 & 1.7 & $\sim 100$ \\
STO on NGO & -8.5 & 16.64 & -7.4 & $\sim 28$ \\
BZC on MgO & -2.12 & 7.31 & -1.1 & $\sim 31$ \\
BZY on BZC-MgO & 2.52 & 11.97 & 2.6 & $\sim 7$ \\
BZC on BZY-MgO & -6.03 & 6.67 & -5.1 & $\sim 2$ \\
SDC on SB-MgO & 1.35 & 10.56 & 1.5 & $\sim 7$ \\
\hline \hline
\end{tabular}

\section{Matthews, Mader, and Light Model}

TABLE III. Fit Parameters for the Matthews, Mader, and Light model [27]. Additionally, the maximal stress value $F$ and the thickness $h_{\max }$ after which the stress decreases are listed.

\begin{tabular}{lrrcrc}
\hline \hline Sample & $c_{2}[\mathrm{GPa}]$ & $b[\mathrm{~nm}]$ & $a[1 / \mathrm{nm}]$ & $F[\mathrm{GPa}]$ & $h_{\max }[\mathrm{nm}]$ \\
\hline SDC on NGO & 6.31 & 13.64 & $\gg 100$ & 1.7 & $\sim 100$ \\
STO on NGO & -23.11 & 6.12 & 0.945 & -7.4 & $\sim 28$ \\
BZC on MgO & -5.76 & 2.69 & $\gg 100$ & -1.1 & $\sim 31$ \\
BZY on BZC-MgO & 6.52 & 4.85 & 0.129 & 2.6 & $\sim 7$ \\
BZC on BZY-MgO & 16.40 & 2.49 & $\gg 100$ & -5.1 & $\sim 2$ \\
SDC on SB-MgO & 3.56 & 4.13 & 0.193 & 1.5 & $\sim 7$ \\
\hline \hline
\end{tabular}

\section{Cammarata, Sieradzki, and Spaepen Model}

TABLE IV. Fit parameters for the Cammarata, Sieradzki, and Spaepen model [30]. Additionally, the maximal stress value $F$ and the thickness $h_{\max }$ after which the stress decreases are listed.

\begin{tabular}{lcc}
\hline \hline Sample & $F[\mathrm{GPa}]$ & $d[\mathrm{GPa}] \mathrm{nm}]$ \\
\hline SDC on NGO & 1.7 & 0.8 \\
STO on NGO & -7.4 & 4.8 \\
BZC on MgO & -1.1 & 1.7 \\
BZY on BZC-MgO & 2.6 & 0.7 \\
BZC on BZY-MgO & -5.1 & 0.1 \\
SDC on SB-MgO & 1.5 & 0.6 \\
\hline \hline
\end{tabular}

\section{Beresford, Lynch, and Chason Model}

Equation (A1) was fit to the stress data $h>h_{\max }$. For obtaining the fitting parameters below, starting values of $\alpha=1$, $\kappa^{\prime}=0.1, n=100$, and $\eta^{\prime}=1000$ are used. 
TABLE V. Fit parameters for the Beresford, Lynch, and Chason model [17].

\begin{tabular}{lccccr}
\hline \hline Sample & $a$ & $\kappa^{\prime}$ & $n$ & $\eta^{\prime}$ & $F[\mathrm{GPa}]$ \\
\hline STO on NGO & 1.415 & 0.056 & 113.183 & 2031.1 & -7.4 \\
BZY on BZC-MgO & 7.203 & 0.125 & 208.398 & 1665.3 & 2.6 \\
BZC on BZY-MgO & 5.741 & 0.004 & 8.867 & 2240.7 & -5.1 \\
SDC on SB-MgO & 3.160 & 0.807 & 1201.34 & 1559.9 & 1.5 \\
\hline \hline
\end{tabular}

Slightly different starting values for the fitting parameter result in a different set of fitting parameters, but of similar magnitude and as accurate. For a resulting set of fitting parameters, 3-4 digits after the comma may be necessary for the fit to look accurate. That the fitting of the model to the data here does not give a clear result is underlined by the comparison to a polynomial fit. Fitting the stress data for BZY-BZC$\mathrm{MgO}$, for example, with the polynomial $a+b x+c x^{2}+d x^{3}$,

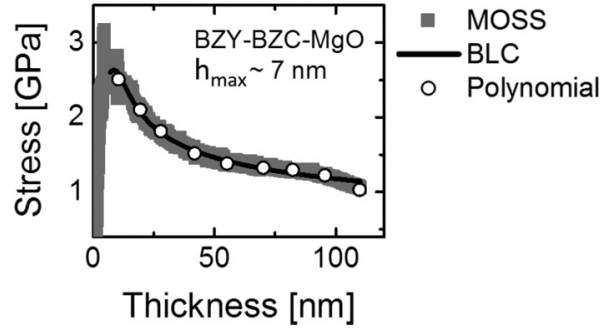

FIG. 7. BLC model and third-order polynomial fit. The BLC model does not give a better fit than a polynomial with the same number of variables.

i.e., with the same number of fitting parameters as the BLC model, yields $a=3.14743 \mathrm{GPa}, b=-0.0707664 \mathrm{GPa}, c=$ $0.000938759 \mathrm{GPa}$, and $d=-4.28076 \times 10^{-6} \mathrm{GPa}$. The BLC model and the polynomial fit are compared for this example in Fig. 7.
[1] R. Ramesh and N. A. Spaldin, Multiferroics: Progress and prospects in thin films, Nat. Mater. 6, 21 (2007).

[2] C. Escorihuela-Sayalero, O. Dieguez, and J. Iniguez, Strain Engineering Magnetic Frustration in Perovskite Oxide Thin Films, Phys. Rev. Lett. 109, 247202 (2012).

[3] F. Y. Bruno, K. Z. Rushchanskii, S. Valencia, Y. Dumont, C. Carretero, E. Jacquet, R. Abrudan, S. Bluegel, M. Lezaic, M. Bibes, and A. Barthelemy, Rationalizing strain engineering effects in rare-earth nickelates, Phys. Rev. B 88, 195108 (2013).

[4] A. Walsh, C. R. A. Catlow, K. H. L. Zhang, and R. G. Egdell, Control of the band-gap states of metal oxides by the application of epitaxial strain: The case of indium oxide, Phys. Rev. B 83, 161202 (2011).

[5] J. S. Kim, G. N. Tam, and G. R. Stewart, Unusual sensitivity of superconductivity to strain in iron-based 122 superconductors, Phys. Rev. B 91, 144512 (2015).

[6] B. Burganov, C. Adamo, A. Mulder, M. Uchida, P. D. C. King, J. W. Harter, D. E. Shai, A. A. Gibbs, A. P. Mackenzie, R. Uecker, M. Bruetzam, M. E. Beaslex, C. J. Fennie, D. G. Schlom, and K. M. Shen, Strain Control of Fermiology and Many-Body Interactions in Two-Dimensional Ruthenates, Phys. Rev. Lett. 116, 197003 (2016).

[7] S. Li, X. Ding, J. Ren, X. Moya, J. Li, J. Sun, and E. K. H. Salje, Strain-controlled thermal conductivity in ferroic twinned films, Sci. Rep. 4, 6375 (2014).

[8] B. Yildiz, "Stretching" the energy landscape of oxides - Effects on electrocatalysis and diffusion, MRS Bull. 39, 147 (2014).

[9] A. Bardal, Th. Matthee, J. Wecker, and K. Samwer, Initial stages of epitaxial growth of Y-stabilized $\mathrm{ZrO}_{2}$ thin films on $a-\mathrm{SiO}_{x} / \mathrm{Si}(001)$ substrates, J. Appl. Phys. 75, 2902 (1994).

[10] T.-B. Hur, Y.-H. Hwang, H.-K. Kim, and H.-L. Park, Study of the structural evolution in $\mathrm{ZnO}$ thin film by in situ synchrotron x-ray scattering, J. Appl. Phys. 96, 1740 (2004).

[11] C. Merckling, M. El-Kazzi, G. Delhaye, V. Favre-Nicolin, Y. Robach, M. Gendry, G. Grenet, G. Saint-Girons, and G. Hollinger, Strain relaxation and critical thickness for Epitaxial
$\mathrm{LaAlO}_{3}$ thin films grown on $\mathrm{SrTiO}_{3}(001)$ substrates by molecular beam epitaxy, J. Cryst. Growth 306, 47 (2007).

[12] G. Niu, G. Saint-Girons, B. Vilquin, G. Delhaye, J.-L. Maurice, C. Botella, Y. Robach, and G. Hollinger, Molecular beam epitaxy of $\mathrm{SrTiO}_{3}$ on $\mathrm{Si}$ (001): Early stages of the growth and strain relaxation, Appl. Phys. Lett. 95, 062902 (2009).

[13] Y. R. Li, Z. Liang, Y. Zhang, J. Zhu, S. W. Jiang, and X. H. Wei, Growth modes transition induced by strain relaxation in epitaxial $\mathrm{MgO}$ thin films on $\mathrm{SrTiO}_{3}(001)$ substrates, Thin Solid Films 489, 245 (2005).

[14] R. People and J. C. Bean, Calculation of critical layer thickness versus lattice mismatch for $\mathrm{Ge}_{x} \mathrm{Si}_{1-x} / \mathrm{Si}$ strained-layer heterostructures, Appl. Phys. Lett. 47, 322 (1985).

[15] J. Tersoff, Dislocations and strain relief in compositionally graded layers, Appl. Phys. Lett. 62, 693 (1993).

[16] L. B. Freund, A criterion for arrest of a threading dislocation in a strained epitaxial layer due to an interface misfit dislocation in its path, J. Appl. Phys. 68, 2073 (1990).

[17] R. Beresford, C. Lynch, and E. Chason, Kinetics of dislocationmediated strain relaxation in InGaAs/GaAs heteroepitaxy, J. Cryst. Growth 251, 106 (2003).

[18] P. L. Gourley, I. J. Fritz, and L. R. Dawson, Controversy of critical layer thickness for InGaAs/GaAs strained-layer epitaxy, Appl. Phys. Lett. 52, 377 (1988).

[19] R. Hull and J. Bean, In situ observations of misfit dislocations in lattice-mismatched epitaxial semiconductor heterostructures, MRS Bull. 19, 32 (1994).

[20] B. Yarlagadda, A. Rodriguez, P. Li, B. I. Miller, F. C. Jain, and J. E. Ayers, Elastic strains in heteroepitaxial $\mathrm{ZnSe}_{1-x} \mathrm{Te}_{x}$ on InGaAs/InP (001), J. Electron. Mater. 35, 1327 (2006).

[21] J. Y. Tsao and B. W. Dodson, Excess stress and the stability of strained heterostructures, Appl. Phys. Lett. 53, 848 (1987).

[22] J. E. Ayers, Heteroepitaxy of Semiconductors: Theory, Growth, and Characterization (CRC Press/Taylor and Francis Group, Boca Raton, FL, 2007).

[23] E. A. Fitzgerald, Dislocations in strained-layer epitaxy: Theory, experiment, and applications, Mater. Sci. Rep. 7, 87 (1991). 
[24] U. W. Pohl, Epitaxy of Semiconductors - Introduction to Physical Principles (Springer-Verlag, Berlin, 2013).

[25] M. Hanbuecken, Stress and Strain in Epitaxy: Theoretical Concepts, Measurements and Applications (Elsevier, Amsterdam, 2001).

[26] J. W. Matthews and A. E. Blakeslee, Defects in epitaxial multilayers, I. Misfit dislocations, J. Cryst. Growth 27, 118 (1974).

[27] J. W. Matthews, S. Mader, and T. B. Light, Accommodation of misfit across the interface between crystals of semiconducting elements or compounds, J. Appl. Phys. 41, 3800 (1970).

[28] R. C. Cammarata, Surface and interface stress effects in thin films, Prog. Surf. Sci. 46, 1 (1994).

[29] R. C. Cammarata and K. Sieradzki, Effects of Surface Stress on the Elastic Moduli of Thin Films and Superlattices, Phys. Rev. Lett. 62, 2005 (1989).

[30] R. C. Cammarata, K. Sieradzki, and F. Spaepen, Simple model for interface stresses with application to misfit dislocation generation in epitaxial thin films, J. Appl. Phys. 87, 1227 (2000).

[31] F. C. Frank and J. H. van der Merwe, One-dimensional dislocations, II. Misfitting monolayers and oriented overgrowth, Proc. R. Soc. London A 198, 216 (1949).

[32] J. L. Vassent, M. Dynna, A. Marty, B. Gilles, and G. Patrat, A study of growth and the relaxation of elastic strain in $\mathrm{MgO}$ on Fe(001), J. Appl. Phys. 80, 5727 (1996).

[33] T. Wang, K. Ganguly, P. Marshall, P. Xu, and B. Jalan, Critical thickness and strain relaxation in molecular beam epitaxy-grown $\mathrm{SrTiO}_{3}$ films, Appl. Phys. Lett. 103, 212904 (2013).

[34] Y. Yu, X. Zhang, J. J. Yang, J. W. Wang, and Y. G. Zhao, Microstructure and strain relaxation of orthorhombic $\mathrm{TmMnO}_{3}$ epitaxial thin films, J. Cryst. Growth 338, 280 (2012).

[35] C. Peng, J. Melnik, J. Li, J. Luo, A. R. Sanger, and K. T. Chuang, $\mathrm{ZnO}$-doped $\mathrm{BaZr}_{0.85} \mathrm{Y}_{0.15} \mathrm{O}_{3-\delta}$ proton-conducting electrolytes: Characterization and fabrication of thin films, J. Power Sources 190, 447 (2009).

[36] J. Tersoff, Stress-induced roughening in epitaxial growth, Appl. Surf. Sci. 102, 1 (1996).

[37] D. E. Jesson, S. J. Pennycook, J.-M. Baribeau, and D. C. Houghton, Surface stress, morphological development, and dislocation nucleation during strained-layer epitaxy, MRS Online Proc. Libr. 317, 31 (1993).

[38] J. W. Gibbs, The Scientific Papers of J. Willard Gibbs (Longmans, Green and Co., London, 1906).

[39] www.tsstsystems.com

[40] www.k-space.com

[41] L. B. Freund, S. Suresh, L. B. Freund, and S. Suresh, Film Stress and Substrate Curvature: Thin Film Materials (Cambridge University Press, Cambridge, UK, 2004).
[42] A. I. Krivchikov, B. Ya Gorodilov, I. G. Kolobov, A. I. Erenburg, D. I. Savitskii, S. B. Ubizskii, I. M. Syvorotka, and L. O. Vasilechko, Structure, sound velocity, and thermal conductivity of the perovskite $\mathrm{NdGaO}_{3}$, Low Temp. Phys. 26, 370 (2000).

[43] T. J. Ahrens, Mineral Physics and Crystallography: A Handbook of Physical Constants (American Geophysical Union, Washington, DC, 1995).

[44] A. Fluri, D. Pergolesi, V. Roddatis, A. Wokaun, and T. Lippert, In situ stress observation in oxide films and how tensile stress influences oxygen ion conduction, Nat. Commun. 7, 10692 (2016).

[45] A. Fluri, A. Marcolongo, V. Roddatis, A. Wokaun, D. Pergolesi, N. Marzari, and T. Lippert, Enhanced proton conductivity in Y-doped $\mathrm{BaZrO}_{3}$ via strain engineering, Adv. Sci. 4, 1700467 (2017).

[46] E. Fabbri, D. Pergolesi, and E. Traversa, Materials challenges toward proton-conducting oxide fuel cells: A critical review, Chem. Soc. Rev. 39, 4355 (2010).

[47] K. D. Kreuer, Proton-conducting oxides, Annu. Rev. Mater. Res. 33, 333 (2003).

[48] D. Pergolesi, V. Roddatis, E. Fabbri, C. W. Schneider, T. Lippert, E. Traversa, and J. A. Kilner, Probing the bulk ionic conductivity by thin film hetero-epitaxial engineering, Sci. Technol. Adv. Mater. 16, 015001 (2015).

[49] T. Pagnier, I. Charrier-Cougoulic, C. Ritter, and G. Lucazeau, A neutron diffraction study of $\mathrm{BaCe}_{x} \mathrm{Zr}_{1-x} \mathrm{O}_{3}$, Eur. Phys. J.: Appl. Phys. 9, 1 (2000).

[50] G. J. Whaley and P. I. Cohen, Relaxation of strained InGaAs during molecular beam epitaxy, Appl. Phys. Lett. 57, 144 (1990).

[51] V. T. Gillard, W. D. Nix, and L. B. Freund, Role of dislocation blocking in limiting strain relaxation in heteroepitaxial films, J. Appl. Phys. 76, 7280 (1994).

[52] D. Pergolesi, M. Fronzi, E. Fabbri, A. Tebano, and E. Traversa, Growth mechanisms of ceria- and zirconia-based epitaxial thin films and hetero-structures grown by pulsed laser deposition, Mater. Ren. Sust. Energy 2, 1 (2012).

[53] Y. Shi, S. C. Lee, M. Monti, C. Wang, Z. A. Feng, W. D. Nix, M. F. Toney, R. Sinclair, and W. C. Chueh, Growth of highly strained $\mathrm{CeO}_{2}$ ultrathin films, ACS Nano 10, 9938 (2016).

[54] G. Liu, Q. Lei, M. A. Wolak, Q. Li, L.-Q. Chen, Ch. Winkler, J. Sloppy, M. L. Taheri, and X. Xi, Epitaxial strain and its relaxation at the $\mathrm{LaAlO}_{3} / \mathrm{SrTiO}_{3}$ interface, J. Appl. Phys. 120, 085302 (2016).

[55] J. Premper, D. Sander, and J. Kirschner, Cantilever stress measurements for pulsed laser deposition of perovskite oxides at $1000 \mathrm{~K}$ in an oxygen partial pressure of $10^{-4}$ millibars, Rev. Sci. Instrum. 86, 033902 (2015). 